REGARDS

SUR LEECONOMIE ALLEMAND

BULLETIN ECONOMIQUE DU CRAC
Regards sur l'économie allemande

Bulletin économique du CIRAC

108 | 2013

Varia

\title{
Le Mittelstand et son financement
}

\section{Karl-Peter Schackmann-Fallis}

Traducteur : Isabelle Bourgeois

\section{OpenEdition}

\section{Journals}

Édition électronique

URL : http://journals.openedition.org/rea/4525

DOI : $10.4000 /$ rea.4525

ISSN : 1965-0787

\section{Éditeur}

CIRAC

Édition imprimée

Date de publication : 2 avril 2013

Pagination : 5-13

ISSN : 1156-8992

\section{Référence électronique}

Karl-Peter Schackmann-Fallis, "Le Mittelstand et son financement », Regards sur l'économie allemande [En ligne], 108 | avril 2013, mis en ligne le 01 mars 2015, consulté le 10 décembre 2020. URL : http:// journals.openedition.org/rea/4525; DOI : https://doi.org/10.4000/rea.4525 


\section{Le Mittelstand et son financement}

\section{Karl-Peter Schackmann-Fallis}

En Allemagne, pas un discours de politique économique n'est prononcé sans que soit invoqué ce Mittelstand, moteur de croissance et facteur de compétitivité. Pour les statisticiens, ce terme désigne les petites et moyennes entreprises employant jusqu'à 500 salariés. Or dans la réalité, il recouvre une approche spécifique de l'entrepreneuriat où se conjuguent autonomie statutaire et commerciale, responsabilité du patron-propriétaire, innovation, et une stratégie de croissance assise sur des bases solides et orientée sur le long terme. Ces PME du Mittelstand ne sont pas l'apanage des grandes villes ou des régions à forte concentration urbaine, elles sont omniprésentes sur le territoire allemand, et $90 \%$ d'entre elles sont des familiales (IfM Bonn). Voilà pourquoi - que ce soit par leur nombre ou pour des raisons culturelles - les PME sont un acteur et un facteur structurel d'une importance primordiale pour l'économie allemande.

Elles ont confirmé leur rôle-clé depuis la profonde récession qu'a connue l'Allemagne à la suite de la crise de la finance mondiale. Non seulement elles ont alors tiré la croissance, mais elles ont aussi stabilisé l'emploi (voir REA 101/ 2011). Il est vrai que l'accès au crédit bancaire leur est plus facile qu'à leurs homologues dans la zone Euro, pour des raisons tant structurelles (liens de partenariat privilégié avec les caisses d'épargne allemandes) que conjoncturelles (taux d'intérêt historiquement bas actuellement). Mais le plus étonnant en comparaison européenne est que les PME du Mittelstand disposent désormais d'un ratio de fonds propres comparable à celui des grandes entreprises. C'est ce qu'il ressort de la dernière édition de l'enquête "Diagnose Mittelstand » publiée le 6 février 2013 par la Fédération des caisses d'épargne allemandes (Deutscher Sparkassen- und Giroverband, DSGV). Elle analyse l'évolution des pratiques à la fois du point de vue des entreprises et de celui de leurs partenaires établis que sont les Sparkassen.

On dénombre 3,7 millions d'entreprises en Allemagne. 99,6\% d'entre elles ont moins de 500 salariés et un CA inférieur à 50 millions $€$ : ce sont ces PME qui constituent le Mittelstand. Elles emploient $60 \%$ des salariés occupant un emploi soumis à cotisations sociales et forment $83 \%$ des apprentis (système dual), selon les données de la plateforme centralisée pour l'enregistrement des entreprises (Unternehmensregister, 2010). A cela s'ajoutent 4,4 millions de travailleurs indépendants (11\% des actifs).

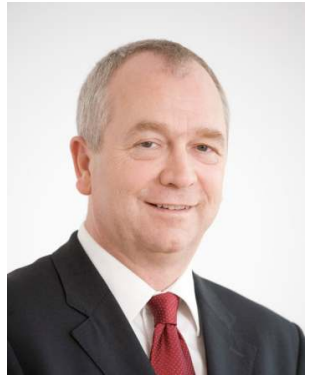

Karl-Peter Schackmann-Fallis, économiste et docteur en sciences politiques, est président du bureau exécutif du Sparkassen- und Giroverband (DSGV) et président de la Direction Economie et Politique.

\section{« PME » ou Mittelstand? Plusieurs définitions}

La définition d'Eurostat est strictement quantitative : effectif inférieur à 250 salariés et CA inférieur à 50 millions $€$.

La définition de l'Institut für Mittelstandsforschung (IfM, Bonn) a deux volets. L'un, quantitatif : effectif inférieur à 500 salariés, CA inférieur à 50 millions $€$. L'autre, qualitatif : le mode de gouvernance qu'induit la propriété ou le contrôle du capital (généralement la famille fondatrice). Dans cette acception, une entreprise du Mittelstand est toujours « dirigée par le propriétaire " (Inhabergeführt), ce qui la distingue de toutes les autres, "dirigées par des managers » extérieurs (Managergeführt). Le pivot de cette gouvernance est l'orientation sur le long terme; elle repose sur des valeurs érigées en normes de droit (voir Bourgeois, 2010) : la quête permanente d'équilibre entre droits et devoirs, intérêts particuliers et collectifs, dont les principes sont inscrits dans la Constitution (art. 14 de la Loi fondamentale : " propriété oblige »). (IB)

L'Allemagne se distingue des autres pays européens par trois particularités. On y compte deux fois plus (16,3\%) d'entreprises occupant entre 10 et 249 sala- 
riés que dans la moyenne de l'UE 27 (7,6\%; 6,7\% en France). Ces entreprises petites et moyennes contribuent à hauteur de $36 \%$ à la création de richesse, alors que cette part n'est que de $30 \%$ en moyenne européenne. Enfin, l'Allemagne - une économie restée industrielle - se distingue par la forte présence de PME dans les secteurs high-tech de l'industrie: leur part y est de $18 \%$ contre $12 \%$ dans l'UE 27 (Eurostat SBA Fact Sheets, 2012).

\section{L'enquête Diagnose Mittelstand}

Publiée tous les ans en début d'année depuis 2002 par la Fédération des caisses d'épargne (Deutscher Sparkassen und Giroverband: DSGV), l'enquête Diagnose Mittelstand repose sur deux volets : l'analyse des bilans des PME clientes et un sondage des chargés de clientèle des Sparkassen comme des banques publiques régionales (Landesbanken; pour plus d'informations sur ce réseau d'établissements, voir REA 88/2008 et 101/2011). Analyse et sondage sont réalisés au dernier trimestre de l'année précédente.

Ainsi, pour l'édition 2013 ont été analysés 123000 bilans anonymisés (exercice 2011) reçus, soit environ la moitié des bilans collectés tous les ans dans la banque de données du groupe DSGV. Ces données sont ensuite complétées et actualisées après le retour des bilans manquants (le taux de retour se situe traditionnellement à $80 \%$ ). Les bilans sont triés notamment selon le système de classification des secteurs d'activité en vigueur en Allemagne (WZ 2008), ce qui permet une lecture fine de l'évolution des entreprises selon leur branche. Les dernières données complètes portent sur l'année 2010 (267 000 bilans).

La présentation de l'analyse des bilans privilégie la valeur médiane. Celle-ci est en effet mieux adaptée pour refléter dans les statistiques une réalité très disparate qu'une valeur moyenne qui, au contraire lisse les disparités existantes. Autrement dit, la médiane permet de mieux cerner ce qui est 'typique' pour telle ou telle branche ou taille d'entreprise en son sein. Un ratio de capitaux propres d'une valeur médiane de 19,8\% signifie ainsi que la moitié des entreprises se situe en dessous de ce seuil ou l'atteint ; l'autre moitié le dépasse. L'enquête recourt également à la valeur 'point zéro' : celle-ci indique combien d'entreprises (en \%) présentent une valeur inférieure ou égale à zéro pour chacun des postes considérés. Ainsi, par exemple, en ce qui concerne les capitaux propres, un taux point zéro de $23,7 \%$ signifie que $23,7 \%$ des entreprises ne disposent pas de capitaux propres, voire que leur bilan est déficitaire.

L'analyse des bilans est complétée par un sondage réalisé parmi les chargés de clientèle du groupe, qui permet de dresser un état des lieux actuel et d'établir des prévisions pour l'année qui suit. Dans le cadre de l'édition 2013 de la Diagnose Mittelstand, 342 Sparkassen (sur les 423 existantes) ont participé à l'enquête. Comme elles sont réparties sur l'ensemble du territoire, leurs réponses sont représentatives.

De son côté, depuis dix ans, la banque publique KfW publie tous les ans en fin d'année les résultats de sa propre enquête sur le financement des entreprises et leurs activités: le $\boldsymbol{K} \boldsymbol{f} \boldsymbol{W}$ - $\boldsymbol{M}$ ittelstandspanel. Réalisée en coopération avec une vingtaine de fédérations professionnelles, cette enquête repose sur leurs analyses en ce qui concerne l'accès au crédit bancaire des entreprises et leurs pratiques en la matière. Le panel des entreprises analysées comprend 3400 sociétés, de toute taille, de tout statut juridique et issues de toutes les branches. Si le KfW-Mittelstandspanel, qui s'appuie par ailleurs sur les données de Destatis (emploi, export...), est lui aussi représentatif au plan macro-économique, il est donc établi sur d'autres bases que l'enquête Diagnose Mittelstand qui entre plus profondément dans les pratiques de la clientèle des caisses d'épargne allemandes. Ces deux rapports sont donc en ce sens complémentaires.

Un ratio de fonds propres en hausse continue...

... et qui atteint les standards internationaux

\section{L'analyse des bilans révèle la robuste santé des PME}

La santé du Mittelstand allemand est robuste. La tendance à la hausse du ratio de fonds propres, qui s'observe continûment tout au long des enquêtes Diagnose Mittelstand menées depuis plus d'une décennie, s'est poursuivie sans interruption en 2011 aussi. La valeur médiane de ce ratio se situe désormais à $19,8 \%$ (contre 16,9 \% en 2010). Chez les TPE, moins richement dotées en capitaux que les plus grandes entités de par leurs activités ou la taille du marché qu'elles desservent (artisanat, marché de proximité...), cette médiane s'est accrue de près de 5 points de pourcentage en l'espace de deux ans pour atteindre aujourd'hui 14,2\%. Quant aux entreprises est-allemandes, leur ratio de fonds propres est maintenant supérieur à celui de leurs homologues de l'ouest : il est de 23,9\%, toutes catégories confondues. Cette part peut étonner, mais elle reflète en réalité une différence dans 'l'allocation' du capital. Généralement, les petites entreprises ont moins de fonds propres du fait de leur statut juridique : ce sont des sociétés de personnes dont le capital appartient au propriétaire qui est garant sur ses propres deniers (persönlich haftender Gesellschafter) ; le capital n'apparaît donc pas dans le bilan. Or dans l'est de l'Allemagne, où les patrons-propriétaires ont (pour l'instant) moins de patrimoine à placer dans leur entreprise, les bilans contiennent plus de fonds propres.

Dans les années 1990 encore, près de la moitié des PME était totalement dépourvues de fonds propres. Aujourd'hui, moins d'un quart d'entre elles sont dans ce cas, et il s'agit alors essentiellement de petites sociétés de personnes 
où le propriétaire est garant de l'entreprise sur ses propres deniers. II y a dix ans encore, les premières vagues de l'enquête Diagnose Mittelstand faisaient apparaître des valeurs médianes de 2 à $4 \%$ - un ordre de grandeur qui prend aujourd'hui des allures 'préhistoriques'. Car depuis, le ratio de fonds propres des PME tend à se rapprocher de plus en plus de celui des grandes entreprises. Sur ce point, l'Allemagne atteint désormais les standards internationaux.

Evolution des fonds propres des PME allemandes 2009-2011 (en \%)

\begin{tabular}{|cccc|}
\hline $\begin{array}{c}\text { Petites entreprises } \\
\text { CA } \leq 1 \text { million } €\end{array}$ & $\mathbf{2 0 0 9}$ & $\mathbf{2 0 1 0}$ & $\mathbf{2 0 1 1}$ \\
$\begin{array}{c}\text { Moyennes entreprises } \\
\text { CA de } 1 \text { à }<50 \text { millions } € \\
\text { Ensemble du Mittelstand } \\
\quad \text { CA de } 0 \text { à } 50 \text { millions } €\end{array}$ & 9,3 & 11,4 & 14,2 \\
$\begin{array}{c}\text { Grandes entreprises } \\
\text { CA }>50 \text { millions } €\end{array}$ & 19,5 & 20,9 & 22,5 \\
\hline
\end{tabular}

Source : DSGV, Diagnose Mittelstand 2013.

Cette tendance au long cours est d'une part le résultat de l'évolution du cadre réservé aux activités. Au début du millénaire ont par exemple été adoptés de nouveaux critères de solvabilité (accords de "Bâle II ») et un régime fiscal moins contraignant pour les capitaux propres. Dans le même temps, l'action concertée du gouvernement fédéral comme de l'ensemble des acteurs du système bancaire allemand dans l'accompagnement des changements structurels auxquels était alors confronté le Mittelstand a créé un environnement porteur (pérennisation du principe de la « banque-maison » et création d'une banque dédiée aux PME auprès de la KfW ; voir REA 49/2000 et 57/2002). D'autre part, cette hausse du ratio de fonds propres est aussi le reflet de l'excellente rentabilité d'une grande partie du tissu productif allemand au cours de ces dernières années, surtout durant les années de croissance, qui a permis aux entreprises de thésauriser en conséquence une partie des bénéfices réalisés.

Les PME allemandes ont une très bonne rentabilité, comme le montre l'analyse des autres postes de leurs bilans. Le ratio résultat d'exploitation/rendement total a atteint en 2010 le niveau record de 7,4\% en valeur médiane, ce qui révèle l'excellente tenue de la rentabilité de l'ensemble des PME depuis l'embellie conjoncturelle et le retour de la croissance. C'est le commerce qui a réalisé les marges les plus faibles : la valeur médiane y était de 4,7\% en 2010 et de 4,2\% en tendance en 2011 ; cela s'explique par son positionnement sur une chaîne de création de valeur relativement réduite.

Evolution du ratio bénéfice net/CA des PME allemandes 2009-2011 (en \%)

\begin{tabular}{|cccc|}
\hline & $\mathbf{2 0 0 9}$ & $\mathbf{2 0 1 0}$ & $\mathbf{2 0 1 1}$ \\
$\begin{array}{c}\text { Petites entreprises } \\
\text { CA } \leq 1 \text { million } €\end{array}$ & 11,1 & 12,2 & 12,8 \\
$\begin{array}{c}\text { Moyennes entreprises } \\
\text { CA de } 1 \text { à }<50 \text { millions } €\end{array}$ & 4,2 & 4,7 & 4,6 \\
$\begin{array}{c}\text { Ensemble du Mittelstand } \\
\quad \text { CA de } 0 \text { à } 50 \text { millions } €\end{array}$ & 6,8 & 7,4 & 6,5 \\
$\begin{array}{c}\text { Grandes entreprises } \\
\text { CA }>50 \text { millions } €\end{array}$ & 1,5 & 2,2 & 2,2 \\
\hline
\end{tabular}

Source : DSGV, Diagnose Mittelstand 2013.

Pour l'ensemble du Mittelstand, la valeur médiane du ratio bénéfice net/chiffre d'affaires devrait se situer à 6,5\% en tendance en 2011. Son recul actuel tient selon toute vraisemblance à la composition très lacunaire encore des bilans mis à la disposition par les PME pour l'édition 2013 de l'enquête Diagnose Mittelstand ; tous n'ont en effet pas encore été transmis (les calculs ultérieurs avaient révélé le même phénomène de sous-estimation lors de la publication de l'enquête 2010). Les bilans qui manquent pour 2011 sont surtout ceux des entreprises au CA inférieur ou égal à 1 million $€$. Or, ces dernières se caractérisent
Un environnement porteur et une excellente rentabilité

Des bilans globalement positifs : Un ratio résultat d'exploitation/ rendement total de $7,4 \%$

Un ratio bénéfice net/CA $(6,5 \%)$ en léger recul 
Les trois quarts au moins des PME sont bénéficiaires

Rémunération du capital tota stable dans l'industrie...

... mais globalement tassée par les faibles taux d'intérêts actuels par un ratio bénéfice net/CA particulièrement élevé : il est de 12,8 \% en 2011 en l'état actuel des calculs. Et cette catégorie a également enregistré la plus forte progression par rapport à 2010 , où ce ratio n'atteignait encore que $12,2 \%$.

Si ces bilans attendus révèlent eux aussi une rentabilité hors pair, alors la valeur médiane définitive pour l'ensemble du Mittelstand devrait être légèrement supérieure encore aux $6,5 \%$ prévisionnels présentés. Mais même si cette valeur estimée demeurait inchangée, elle n'en resterait pas moins excellente : elle est en effet nettement supérieure à la moyenne de ces vingt dernières années, où elle oscillait entre 3,4 \% (1995) et 5,6 \% (2004). Les PME allemandes se caractérisent par une solide rentabilité.

Un autre indice vient à l'appui de ce constat : quelle que soit la taille des entreprises considérées, la part de celles qui réalisent des bénéfices n'a cessé de progresser. A l'inverse, en 2011, la part des PME déficitaires est tombée à un niveau historiquement bas depuis qu'existe l'enquête Diagnose Mittelstand: elle atteignait $17,2 \%$ seulement.

Evolution de la part des PME allemandes excédentaires/déficitaires 2009-2011 (en \%)

\begin{tabular}{|ccccccc|}
\hline & \multicolumn{2}{c}{2009} & \multicolumn{2}{c}{$\mathbf{2 0 1 0}$} & \multicolumn{2}{c|}{$\mathbf{2 0 1 1}$} \\
& Excéd. & Déficit & Excéd. & Déficit & Excéd. & Déficit \\
$\begin{array}{c}\text { Petites entreprises } \\
\text { CA } \leq 1 \text { million } €\end{array}$ & 79,1 & 20,9 & 80,6 & 19,4 & 81,0 & 19,0 \\
$\begin{array}{c}\text { Moyennes entreprises } \\
\text { CA de 1 à }<50 \text { millions } €\end{array}$ & 79,7 & 20,3 & 83,0 & 17,0 & 84,1 & 15,9 \\
$\begin{array}{c}\text { Ensemble du Mittelstand } \\
\quad \text { CA de 0 à 50 millions } €\end{array}$ & 79,3 & 20,7 & 81,7 & 18,3 & 82,8 & 17,2 \\
$\quad \begin{array}{c}\text { Grandes entreprises } \\
\text { CA }>50 \text { millions } €\end{array}$ & 70,7 & 29,3 & 77,0 & 23,0 & 77,1 & 22,9 \\
\hline
\end{tabular}

Source : DSGV, Diagnose Mittelstand 2013.

De 2009 à 2010, si la rémunération du capital total a augmenté elle aussi pour atteindre $12,5 \%$, elle devrait toutefois avoir légèrement reculé en tendance en 2011 et se situer à 11,6 \% en valeur médiane pour l'ensemble des PME selon les calculs actuels. Mais cette évolution varie largement selon les branches. Ainsi, dans l'industrie, les PME sont parvenues à stabiliser à peu près la rentabilité de leur capital total, alors que les grandes entreprises ont pu, elles, l'accroître. C'est surtout dans le secteur des services que la rémunération du capital enregistre en 2011 un recul par rapport à 2010. La même tendance s'observe dans le commerce et le BTP, quoique moins prononcée.

Certes, ici aussi, les vagues précédentes de l'enquête ont montré que la valeur définitive, obtenue une fois que tous les bilans d'entreprise ont pu être analysés, est généralement un peu plus élevée que ne le laissent penser les valeurs prévisionnelles ; celles-ci sont donc probablement sous-évaluées. Néanmoins, un léger recul du coefficient de rendement du capital semble vraisemblable sous l'effet de la longue période de faible taux d'intérêt que nous traversons actuellement. Cela est particulièrement net en ce qui concerne le seuil de rendement marginal en dessous duquel les entreprises investissent dans le renouvellement des immobilisations corporelles.

Les faibles taux d'intérêt ont en effet un impact direct sur le financement de l'économie réelle en Allemagne. Durant la phase de récession après 2008, nombreuses ont été les grandes banques commerciales à se retirer de l'activité de financement des entreprises. Et s'il n'y a pas eu alors de credit crunch, c'est que d'autres établissements, principalement les caisses d'épargne (Sparkassen) et les banques coopératives (Genossenschaftsbanken) y ont suppléé. Aujourd'hui assainies, les grandes banques commerciales sont à nouveau présentes sur le marché du crédit aux entreprises, et l'offre à destination du Mittelstand est quasi pléthorique. La concurrence acharnée au sein du secteur bancaire allemand crée, pour les petites et moyennes entreprises, des conditions actuellement plus que favorables. 
Or si, à court terme, le faible coût lié au recours à des capitaux extérieurs accroît la rentabilité des entreprises, à long terme, en revanche, le rendement des actifs immobilisés s'en trouvera réduit d'autant. En effet, lorsque les intérêts des capitaux empruntés sont bas, les entreprises réalisent plus de projets d'investissement tout juste rentables à ces conditions - mais qui ne le seraient pas en cas de taux élevés. II faut donc s'attendre à ce que, à long terme, cette situation ne soit pas sans effet sur la rémunération du capital des entreprises.

Le ratio masse salariale/CA est resté stable dans la catégorie des entreprises réalisant un CA annuel de plus de 1 million $€$ : il est de 22,0 \% en 2012 contre $22,1 \%$ en 2011. Globalement, dans les PME réalisant un CA allant jusqu'à 50 millions $€$, ce ratio se situe à $18,7 \%$ en 2012 (18,2 \% en 2011). Si ce ratio a par moments dépassé les $22 \%$ en moyenne dans l'ensemble du Mittelstand, il n'a cependant que peu varié en tendance depuis les années 1990.

Du fait que de nombreuses branches se caractérisent par une situation proche du plein emploi, la progression des salaires a été plus dynamique en 2011 que par le passé. La hausse de la productivité compensant actuellement cette évolution, la compétitivité-prix des entreprises allemandes ne s'en trouve donc pas affectée.

\section{Prévisions 2013 des Sparkassen : le Mittelstand anticipe la reprise conjoncturelle}

La crise de la dette européenne suscite de profondes inquiétudes dans le Mittelstand. En effet, bien que l'économie allemande se présente comme particulièrement robuste en comparaison des problèmes colossaux que rencontrent celles des pays en proie à la crise, elle n'en a pas moins subi les répercussions. C'est ainsi que le PIB allemand a lui aussi baissé au cours du quatrième trimestre 2012.

Nombreuses sont donc les branches où l'inquiétude face à l'évolution future de la crise a amené les entreprises à ajourner leurs projets d'investissement. Dans un tel contexte, il est rassurant de constater que le Mittelstand allemand a accumulé des réserves de capitaux propres d'une ampleur inconnue jusqu'ici. Et, selon les prévisions des Sparkassen, cette tendance est amenée à se poursuivre, voire à se développer encore, surtout dans le Bade-Wurtemberg où $75 \%$ des caisses d'épargne ont constaté une hausse des capitaux propres par rapport à 2011. Il est en effet impossible d'identifier une quelconque tendance au recours accru à des capitaux étrangers (à des taux particulièrement intéressants actuellement) en lieu et place des fonds propres.

En ce qui concerne les entrées de commandes et le chiffre d'affaires, la plupart des caisses d'épargne constatent à la fin 2012 chez leurs PME clientes une situation " inchangée ». L'évaluation des situations aux deux bouts de l'échelle les amène à des taux largement équivalents : 18,1\% pour une situation « plutôt meilleure " et $21,3 \%$ à l'inverse pour une situation " plutôt moins bonne ». Ces niveaux sont loin de traduire un quelconque risque de récession. Mais il existe de nettes disparités régionales. C'est dans le Mecklembourg que les évaluations sont les plus optimistes : la moitié des caisses d'épargne y relève la poursuite de l'amélioration de la situation de ses entreprises clientes; pas une seule n'a décelé une détérioration. A l'opposé, en Saxe, un Land qui se caractérise par une industrie largement tournée vers l'export, les PME sont nettement plus affectées par les turbulences sur le marché mondial : $45 \%$ des caisses d'épargne du Land y relèvent une détérioration.

Au dernier trimestre 2012, l'investissement a reculé en Allemagne, ce qui se traduit dans les résultats de l'enquête Diagnose Mittelstand qui avait été réalisée au cours des derniers mois de l'année. Dans l'ensemble, les Sparkassen s'attendent donc à une évolution en termes constants en matière de finance-
Le ratio masse salariale/CA est fonction de la taille de l'entreprise

La crise de la dette inquiète le Mittelstand...

... qui constitue des réserves de fonds propres

Des difficultés à l'export, mais pas de récession en vue

Léger recul de la demande de crédits 
Les PME investissent pour étendre leurs capacités
Groupe Sparkassen : $40 \%$ des PME clientes

ment des investissements, le volume des crédits octroyés ne se trouvant pas limité par une offre déficiente, mais par une demande en léger recul.

En revanche, en comparaison avec 2011, la structure des crédits d'investissement s'est modifiée. La fréquence avec laquelle les entreprises réalisant des immobilisations corporelles avancent comme motif premier l'investissement dans le but d'étendre leurs capacités est en recul : après avoir atteint un pic momentané de $35 \%$ en 2011 , elle est retombée à $24,7 \%$, c'est-à-dire son niveau normal.

Réactions des PME clientes des Sparkassen en cette période de faibles taux d'intérêt (en \% de l'ensemble des réponses ; 2 réponses au maximum par Sparkasse)

\begin{tabular}{|lr|}
\hline Thésaurisation accrue des réserves & 39,4 \\
Plus d'investissements de rationalisation & 22,7 \\
Pas de changement significatif & 15,8 \\
Plus d'investissements dans l'extension des capacités & 13,7 \\
Autres & 6,3 \\
Tendance accrue aux placements à risques & 2,1 \\
\hline
\end{tabular}

Source : DSGV, Diagnose Mittelstand 2013.

La phase particulièrement longue de faibles taux d'intérêts influe largement sur la stratégie du Mittelstand. Près de $40 \%$ des Sparkassen relèvent ainsi que leurs clients thésaurisent plus de liquidités, ce qui se reflète également dans l'encours des établissements, et ce, à l'échelon de l'ensemble de l'Allemagne. La deuxième réaction des PME est de renforcer leurs investissements pour effectuer des rationalisations. C'est ce que constatent près de $23 \%$ des caisses d'épargne. Dans le même temps, $14 \%$ d'entre elles relèvent que leurs clients accroissent leurs investissements pour étendre leurs capacités - autre réaction encore aux taux bas pratiqués. Le fait que, même en phase de tassement passager de la conjoncture européenne à la fin 2012 et malgré un taux normal d'utilisation des capacités de production, les entreprises clientes des Sparkassen ne se contentent pas de maintenir en l'état leurs capacités a de quoi rassurer visiblement, elles anticipent la reprise à venir.

\section{La confiance des PME allemandes repose aussi sur la sécurité que procure un réseau bancaire de proximité}

La sérénité des entreprises du Mittelstand est largement fondée sur les liens de partenariat étroits avec les « banques-maison » (Hausbank) des PME que sont les 422 Sparkassen allemandes. Plus de $40 \%$ des PME sont ainsi clientes de ces caisses d'épargne qui octroient près du quart du crédit bancaire aux entreprises (et une large majorité du crédit aux artisans et aux travailleurs indépendants). Aux Sparkassen s'ajoutent les huit banques régionales (Landesbanken) dont l'activité de crédit s'adresse aux moyennes et grandes entreprises. Leur part de marché en la matière dépasse tant celle des banques coopératives que celle des grandes banques commerciales. Au total, malgré la forte concurrence des banques coopératives au niveau local et des grandes banques commerciales au niveau national, la part de marché de la Sparkassen-Finanzgruppe se situe depuis plusieurs années à quelque $42 \%$, avec une hausse de 3,2\% de celle des seules caisses d'épargne depuis la crise.

Crédits ${ }^{*}$ accordés à l'économie réelle 2008-2012 (en \%)

\begin{tabular}{|c|c|c|c|c|c|}
\hline & 2008 & 2009 & 2010 & 2011 & $2012(P)$ \\
\hline Sparkassen-Finanzgruppe & 42,5 & 43,0 & 43,0 & 42,7 & 42,8 \\
\hline Sparkassen & 24,0 & 25,3 & 26,1 & 26,5 & 27,2 \\
\hline Landesbanken & 18,5 & 17,7 & 16,9 & 16,2 & 15,6 \\
\hline Coopératives de crédit & 14,9 & 16,0 & 16,7 & 17,1 & 18,1 \\
\hline Grandes banques commerciales & 12,7 & 12,1 & 11,4 & 11,1 & 10,8 \\
\hline Banques privées régionales, succursales de banques étrangères & 12,9 & 12,6 & 12,9 & 13,6 & 13,9 \\
\hline Autres & 17,0 & 16,3 & 16,0 & 15,5 & 14,4 \\
\hline
\end{tabular}

Source : Deutsche Bundesbank, calculs propres du DSGV. *) Y compris crédits à l'immobilier locatif ; exceptés les crédits aux institutions financières. 
Or les données chiffrées seules ne suffisent pas à rendre compte ni du modèle économique qui fonde les profonds liens de confiance entre les Sparkassen et le Mittelstand, ni non plus des structures et du mode de fonctionnement sur lequel il repose. Comme le « modèle » du Mittelstand, celui des Sparkassen aussi se caractérise en effet par une culture établie sur des valeurs aujourd'hui transcrites dans le droit. Et le financement des entreprises du Mittelstand fait partie intégrante de la mission que s'était donnée le réseau lors de sa fondation voilà plus de 200 ans et qui perdure jusqu'à aujourd'hui dans une "mission d'intérêt public " (öffentlicher Auftrag) qui légitime le statut juridique particulier des caisses d'épargne allemandes. De ce fait, et contrairement aux banques commerciales, elles ne considèrent pas les entreprises clientes comme de simples emprunteurs, mais comme des partenaires avec qui, dans d'autres domaines d'activité aussi, elles tissent des liens profonds qui se révèlent profitables aux deux parties.

Dans la pratique, le financement des PME se présente par essence comme une activité de proximité. Certes, les 422 Sparkassen partagent la même marque et les mêmes valeurs, mais comme elles sont des entités juridiquement indépendantes, elles agissent en pleine autonomie. Par l'intermédiaire de leurs 15600 filiales, elles sont présentes sur l'ensemble du territoire allemand; il y en a au moins une dans chaque commune. Ces dernières servent exclusivement le marché local où elles sont implantées, conformément au principe de territorialisation de certains services d'intérêt économique général (voir REA 88/2008).

Pour une entreprise, il est donc facile de prendre contact avec la caisse d'épargne du cru. Si la distance géographique est faible, les circuits de décision sont courts eux aussi : la direction de chaque établissement décide seule d'accorder ou de refuser un crédit et porte seule la responsabilité de sa décision. Autrement dit, même un petit établissement avec un total bilan de 300 millions $€$ seulement et 80 salariés pratique le financement des PME. Les caisses d'épargne allemandes sont non seulement proches des entreprises du Mittelstand en ce qui concerne la distance géographique, mais aussi par leur taille comme leurs préoccupations.

II n'y rien de philanthropique dans cette proximité avec le client. Elle est au contraire dans l'intérêt économique bien senti de chaque établissement dont les fonds propres sont alimentés exclusivement par les bénéfices thésaurisés. De ce fait, ils disposent de plusieurs instruments de management du risque, dont deux sont caractéristiques des particularités des Sparkassen. D'une part, la notation interne de la clientèle ne se limite pas à l'analyse des données chiffrées. Elle tient compte aussi de facteurs tels que la compatibilité de sa production avec les normes environnementales, l'expérience managériale de la direction, l'existence ou non de dispositions pour la transmission de l'entreprise ou, plus généralement - et c'est là l'aspect principal - elle prend en considération tous les éléments déterminants pour la pérennité de l'entreprise cliente. D'autre part, pour mieux évaluer l'activité et les perspectives d'une entreprise dans son environnement qu'est la branche, les établissements recourent à la banque de données interne au réseau dont le corpus s'alimente des bilans anonymisés de leurs clients et sur la base duquel sont établies à intervalles réguliers des prévisions conjoncturelles pour plus d'une centaine de secteurs de l'industrie (voir REA 101/2011). Ce savoir, qui permet une analyse fine du risque client, est ensuite en quelque sorte 'restitué' à l'entreprise avec sa notation. C'est sur ces mêmes bases que reposent les enquêtes annuelles Diagnose Mittelstand.

C'est grâce à cette proximité avec le marché et à cette connaissance intime des entreprises clientes que les caisses d'épargne ont pu développer encore en 2012 l'octroi de nouveaux crédits aux entreprises : son volume a atteint 69,9 milliards $€(+4,8 \%$ par rapport à 2011). Au total, à la fin de l'année écoulée, le montant des crédits aux entreprises accordés par le réseau s'élevait à 339,1 milliards $€(+3,8 \%$ par rapport à 2011$)$, soit le niveau le plus haut atteint au cours des quatre années écoulées. Depuis 2007 en effet, l'année précédant
Une culture partagée

Un business model établi sur la proximité avec le client

339 milliards $€$ de crédits aux entreprises en 2012 
Un rôle clé dans le développement économique régional

Les aides de la KfW sont attribuées par les Sparkassen

Sparkassen : un ratio prêts/dépôts de $80 \%$ la crise de la finance mondiale, les Sparkassen ont continûment accru le nombre des nouveaux crédits aux entreprises et aux travailleurs indépendants - et ce, même en 2009, alors que le PIB allemand s'effondrait de près de $5 \%$. Non seulement les PME n'ont alors pas subi de credit crunch, mais elles n'ont cessé d'investir en prévision d'un retour durable de la croissance. Et de fait, en 2012 aussi, la majorité ( 82 \%) des prêts consentis sont des crédits sur le moyen et le long terme, autrement dit : destinés à l'investissement.

Cela étant, le rôle des Sparkassen ne se réduit pas à celui de prêteur. De par leur mission d'intérêt général, elles assument la responsabilité de contribuer au développement économique de leur région ou commune d'implantation. Elles le font en tant qu'employeur ou contribuable, mais aussi en parrainant des projets à dimension sociale. En ce qui concerne le Mittelstand, cette responsabilité implique aussi qu'elles apportent leur soutien à la création d'entreprises et qu'elles mettent en œuvre les programmes publics de soutien en la matière. En 2012, elles ont ainsi co-financé la création de 11000 entreprises (qui ont généré 33000 emplois). A cet effet, elles mettent à disposition des créateurs des collaborateurs spécialisés, voire des plateformes de coaching, et elles ont développé leurs propres outils pour optimiser non seulement leur demande de crédits, mais aussi leur business plan.

Ces créations ont été co-financées pour moitié par des programmes publics et par les Sparkassen qui en assument alors seules le risque. Elles sont en effet le principal partenaire de la banque publique de soutien aux entreprises qu'est la $\mathrm{KfW}$. Cette dernière n'étant pas habilitée à intervenir directement, c'est par les établissements de crédit que transitent les aides, dont les Sparkassen. Concrètement, cela signifie que le dossier de demande d'aide à la création d'entreprise est soumis par le candidat à sa banque ou caisse d'épargne qui l'évalue et, qui en cas d'accord, supporte aussi l'essentiel des risques. Les Sparkassen sont ainsi l'opérateur de plus de $43 \%$ (2012) du volume des programmes de soutien de la KfW aux entreprises du Mittelstand. Connaissance de la donne locale et proximité avec le client favorisent une mise en œuvre efficiente, car sur mesure, des aides publiques. S'il est important, le rôle des Sparkassen en matière de crédit aux entreprises ne se borne cependant pas à cette complémentarité entre l'offre de pogrammes publics et l'octroi de crédits au cas par cas. Car seulement $10 \%$ du volume total de crédits aux entreprises a été attribué dans le cadre de la mise en œuvre de ces programmes.

L'essentiel de ces 339,1 milliards $€$ de crédits (2012) était en effet financé sur fonds propres. Or ces capitaux proviennent des dépôts de la clientèle, en grande partie des particuliers, qui atteignaient 830 milliards $€$ en 2012 . La crise de la finance mondiale a fait oublier quels sont les fondements mêmes du métier de banquier : refinancer le crédit aux entreprises (et aux particuliers) grâce à leurs dépôts. Chez les Sparkassen, le ratio prêts/dépôts (loan to deposit) s'établit à environ $80 \%$ - et ce, depuis longtemps. Et à trop se focaliser aujourd'hui sur les seuls critères quantitatifs que sont les lignes de crédit bancaire, on en néglige trop souvent les aspects qualitatifs du partenariat entre les caisses d'épargne et les entreprises clientes: l'ancrage dans le 'terroir', et le partage d'une culture de gestion prudente et orientée sur le long terme. Le poids économique de ce réseau polycentrique d'établissements réside au fond dans le 'recyclage' de dépôts en crédits - c'est là leur apport au développement économique local dont bénéficie en premier lieu le Mittelstand.

Le Mittelstand eSt 'EN PLeine forme’. Malgré le ralentissement temporaire de la croissance allemande, les fonds propres des PME atteignent aujourd'hui des niveaux historiques; les principaux indicateurs du climat des affaires incitent également à l'optimisme.

Or le contexte européen et international est lui aussi déterminant pour leurs activités. Et on s'aperçoit que, en matière d'investissements, les PME allemandes 
sont restées longtemps en deçà de ce que leur auraient permis ces fonds propres. Au cours des dix premières années du millénaire en effet, environ les deux tiers de l'épargne allemande ont alimenté les flux d'investissement hors des frontières. En d'autres termes, l'Allemagne ne s'est pas contentée d'enregistrer des excédents en matière de biens exportés, mais elle en a également fourni le financement, les capitaux exportés 'recyclant' en quelque sorte les excédents de sa balance commerciale.

Les faibles taux d'intérêts actuels sont un autre facteur déterminant du contexte européen. Or une politique de taux bas installée dans la durée se révèle à double tranchant. D'un côté, elle permet actuellement de mettre facilement à disposition des entreprises des capitaux étrangers. La hausse des investissements d'équipement rendue ainsi possible améliore alors le rapport du facteur capital au facteur travail, et accroît la productivité. C'est là une évolution positive, notamment au vu du vieillissement démographique en Allemagne. D'un autre côté, cette politique incite à des comportements dont les effets peuvent se révéler néfastes à long terme. Les investissements réalisés dans le but de rationaliser la production vont certes, dans un premier temps, accroître la compétitivité de l'entreprise; mais dans un deuxième temps, ces investissements qui n'auraient peut-être pas été rentables à des taux plus élevés risquent de détruire des emplois.

Dans le secteur bancaire allemand, le financement du Mittelstand subit actuellement un nouvel engouement. Comme il est de notorité publique que cette activité ne présente qu'un risque limité, les capitaux affluent dans ce 'havre de stabilité' que sont les PME en Allemagne. Même les grandes banques commerciales qui, pour optimiser leur modèle économique, s'étaient retirées du financement du Mittelstand il y a quelques années seulement, s'apprêtent à se repositionner dans ce métier. Les Sparkassen, pour leur part, ne l'avaient jamais abandonné ; c'est une des raisons pour lesquelles l'Allemagne n'a jamais connu ce credit crunch tant redouté outre-Rhin comme ailleurs. Aujourd'hui, il n'y a plus lieu de débattre d'un quelconque risque de resserrement - bien au contraire, il y a une offre surabondante de crédit bancaire pour les PME.

Une durée persistante de faibles taux d'intérêts n'est donc pas adaptée à l'économie domestique allemande. Or l'Allemagne est insérée par des liens étroits dans un ensemble solidaire qu'est le marché unique et l'union monétaire. Vus sous cet angle, les faibles taux d'intérêts à eux seuls font que l'Allemagne apporte aux Etats membres débiteurs nets une contribution en termes de solidarité et de stabilité.

(Traduction : Isabelle Bourgeois)

\section{Indications bibliographiques}

- Bourgeois I., PME allemandes : les clés de la performance, Editions du CIRAC, 2010

- Bourgeois I., LASSERRE R., " Les PME allemandes : une compétitivité à dimension sociale et humaine », in OSEO, PME 2010 (www.cirac.u-cergy.fr/colloques_etudes_wp/cirac_pme.pdf)

- BRÄMer P., Gischer H., Richter T., « Le système bancaire allemand et la crise financière », Regards sur l'économie allemande, $\mathrm{n}^{\circ} 101$, été 2011

- DSGV, Diagnose Mittelstand 2013 (www.dsgv.de/de/fakten-und-positionen/publikationen/index. html). Une version en langue anglaise est également disponible via : publikationen@dsgv.de

- KFW, KfW-Mittelstandspanel 2012 (www.kfw.de/kfw/de/l/II/Download_Center/Fachthemen/Re search/Studien_und_Materialien/PDF-Dokumente_KfW-Mittelstandspanel/KfW-Mittelstandspanel 2012.pdf). Une version en langue anglaise est également disponible (www.kfw.de/kfw/en/l/II/ Download_Center/Research/PDF-Dateien-Mittelstandspanel/apf.pdf)

- Schackmann-Fallis K.-P., «Au cœur de l'économie réelle: le lien Mittelstand/Sparkassen », Regards sur l'économie allemande, $\mathrm{n}^{\circ} 101$, été 2011

- SchackmanN-FALliS K.-P., "Les Sparkassen, facteur de stabilité pour l'économie allemande », Regards sur l'économie allemande, n 88, octobre 2008

- SchWARTz M., "Kredite an KMU - Schere in Europa geht weiter auf », KfW Economic Research, Fokus Volkswirtschaft, $\mathrm{n}^{\circ} 16$ (22-02-2013). 